\title{
Increased lung neutrophil apoptosis and inflammation resolution in nonresponding
} pneumonia

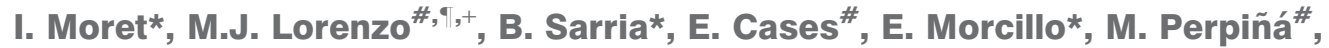 \\ J.M. Molina ${ }^{\S}$ and R. Menéndez ${ }^{\#, f}$
}

ABSTRACT: Neutrophil activation state and its relationship with an inflammatory environment in community-acquired pneumonia (CAP) remain insufficiently elucidated. We aimed to evaluate the neutrophil apoptosis and cytokine pattern in CAP patients after $72 \mathrm{~h}$ of treatment, and their impact on infection resolution.

Apoptosis of blood and bronchoalveolar lavage (BAL) neutrophils was measured in nonresponding CAP (NCAP), in responding CAP (blood only) and in patients without infection (control). Proinflammatory (interleukin (IL)-6, IL-8) and anti-inflammatory (IL-10) cytokines were measured. Main outcomes were clinical stability and days of hospitalisation.

Basal neutrophil apoptosis was higher in the BAL and blood of NCAP, whereas spontaneous apoptosis (after $24 \mathrm{~h}$ culture) was lower. Cytokines in NCAP were higher than in responding CAP and control: IL-6 was increased in BAL and blood, IL-8 in BAL and IL-10 in blood. An increased basal apoptosis $(\geqslant 20 \%)$ in BAL of NCAP was associated with lower systemic IL-10 $(p<0.01)$, earlier clinical stability $(p=0.05)$ and shorter hospital stay $(p=0.02)$. A significant correlation was found for systemic IL- 6 and IL-10 with days to reach stability and length of stay.

After $72 \mathrm{~h}$ of treatment, an increased basal alveolar neutrophil apoptosis might contribute to downregulation of inflammation and to faster clinical stability.

KEYWORDS: Bronchoalveolar lavage, cytokines, length of stay, neutrophil apoptosis, nonresponding community-acquired pneumonia

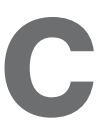
ommunity-acquired pneumonia (CAP) has an incidence of five per 1,000 adults [1] and its mortality ranges between $5 \%$ and $15 \%$ of hospitalised patients. An adequate host response facilitates microbial killing while limiting excessive inflammation and tissue damage [2]. However, $\sim 10 \%$ of CAP patients develop nonresponding $\mathrm{CAP}$ (NCAP), which leads to delayed resolution and poor outcome [3].

In CAP, activated neutrophils in the blood migrate and accumulate in the lungs, ingest and kill bacteria and, finally, undergo apoptosis. Macrophages remove apoptotic neutrophils, precluding subsequent release of inflammatory cytokines and promoting resolution of inflammation [4,5]. Although neutrophils are usually short-lived immune cells, their lifespan prolongation is critical in their efficacy. However, as neutrophils' lytic enzymes can also induce organ damage, their activation and survival must be tightly regulated [5-9].

In NCAP, a persistent lung and systemic inflammation has been reported [10-12]. However, the state of neutrophil activation remains to be elucidated. We hypothesised that the persistence of activated neutrophils in the lungs in NCAP might be contributing to the maintenance of inflammation and to a delayed resolution.

We aimed primarily to evaluate the neutrophil apoptosis and the cytokine pattern (interleukin (IL)-6, IL-8 and IL-10), both in the lung and in blood, in hospitalised patients with NCAP. Secondarily, we investigated their impact on the clinical parameters of stability and length of stay (LOS).

\section{METHODS AND MATERIALS}

\section{Study design}

A prospective study was performed in admitted patients with CAP. Pneumonia was defined as the presence of a new infiltrate with concordant symptoms and laboratory results (fever $\geqslant 38^{\circ} \mathrm{C}$, purulent sputum and leukocyte count $\geqslant 12,000 \mathrm{~mm}^{-3}$ ). Exclusion criteria were admission in the previous 15 days and immunosuppressive treatment.

\section{AFFILIATIONS}

*Pharmacology Dept, School of Medicine, University of Valencia \#Pneumology Service,

${ }^{5}$ Microbiology Service, University and Politecnic Hospital La Fe, + IIS La Fe-Fundación Bancaja, ${ }^{f}$ CIBER de Enfermedades Respiratorias (CIBERES), Valencia, and

"Medicine-Doctoral Programme Dept, Barcelona Autonomous University, Barcelona, Spain.

CORRESPONDENCE

R. Menéndez

Pneumology Service

University and Politecnic Hospital La $\mathrm{Fe}$

Bulevar Sur

46026 Valencia

Spain

E-mail: rmenend@separ.es

Received:

Dec 102010

Accepted after revision:

March 082011

First published online:

March 242011

European Respiratory Journal Print ISSN 0903-1936

Online ISSN 1399-3003 
The inclusion criteria in the NCAP group were: fulfilment of clinical conditions of NCAP after $72 \mathrm{~h}$ of treatment (see definition below) and performance of bronchoscopy indicated by the physician in charge. Patients were included in the study on the day when they underwent bronchoscopy.

Two control groups matched by age $( \pm 10 \mathrm{yrs})$ and comorbid conditions were included: 1) the CAP group comprised patients with CAP who reached clinical stability (definition below); 2) the control group comprised patients without infection referred to bronchoscopy for peripheral lung nodules or minor haemoptysis.

We collected data on age, sex, smoking habits, comorbidities, clinical signs and symptoms, Pneumonia Severity Index [13], chest radiograph, biochemical analyses, microbiological findings, and previous and current antibiotic treatment.

The study was approved by the ethics committee of the University and Politecnic Hospital La Fe (Valencia, Spain) and written informed consent was obtained. No bronchoalveolar lavage (BAL) fluid was obtained in the CAP group because the ethics committee would not approve bronchoscopy in patients with adequate response.

\section{Definitions}

NCAP was defined as persistence of a high temperature $\left(\geqslant 38^{\circ} \mathrm{C}\right)$ and/or clinical symptoms after $72 \mathrm{~h}$ of antibiotic treatment, chest radiographic progression of pneumonia $(>50 \%$ increase of infiltrates along with persistence of high temperature and/or clinical symptoms), empyema, septic shock and/or the need for mechanical ventilation [12]

The CAP group comprised patients who fulfilled clinical stability criteria $[14,15]$. Stability was defined as the achievement of all the following parameters: temperature $\leqslant 37.2^{\circ} \mathrm{C}$, heart rate $\leqslant 100$ beats per $\mathrm{min}$, respiratory rate $\leqslant 24$ breaths per min, systolic blood pressure $\geqslant 90 \mathrm{mmHg}$, and oxygen saturation $\geqslant 90 \%$ or arterial oxygen tension $\geqslant 60 \mathrm{mmHg}$ without supplemental oxygen.

LOS was calculated as the number of days from admission until discharge.

\section{Obtaining and processing of BAL}

BAL was performed according to recommended guidelines [16]. In the NCAP group, BAL was performed in the involved lobe, and in those patients with diffuse pulmonary infiltrates or in the control group, in the middle lobe or lingula. Five aliquots of sterile saline solution were instilled and immediately aspirated. The first aliquot $(20 \mathrm{~mL})$ was discarded. The remaining four aliquots (30 mL each) were pooled together in a single siliconised sterile glass: half of these four aliquots were sent to a microbiology laboratory and the other half to a biochemistry laboratory. The mean \pm SEM volume of BAL fluid obtained was $39 \pm 3 \mathrm{~mL}$

\section{Neutrophil purification from BAL samples}

The BAL samples were immediately pelleted by centrifugation at $353 \times g$ for $5 \mathrm{~min}$ at $4{ }^{\circ} \mathrm{C}$. The supernatant volume was measured and frozen. The pellet was resuspended with culture medium (RPMI 1640 without phenol red; Invitrogen, Carlsbad, CA, USA), passed through two sheets of gauze, and centrifuged at $353 \times g$ for $5 \mathrm{~min}$ at $4{ }^{\circ} \mathrm{C}$. The cell pellet was washed twice with PBS and resuspended with hypotonic solution $\left(\mathrm{NH}_{4} \mathrm{Cl} 155 \mathrm{mM}, \mathrm{NaHCO}_{3} 2.46 \mathrm{mM}\right.$, EDTA·4H $\mathrm{H}_{2} \mathrm{O} 3.72 \mathrm{mM}$ $\mathrm{pH}$ 7.4) for $5 \mathrm{~min}$ on ice. After centrifugation at $304 \times g$ for $5 \mathrm{~min}$ at $4^{\circ} \mathrm{C}$, the pellet was resuspended in PBS. The neutrophil amount and viability were determined using a trypan blue exclusion method $[17,18]$. For light microscopy studies, cytospin slides were stained by the Panoptic method (Panreac Quimica SA, Barcelona, Spain).

\section{Preparation of blood samples}

In NCAP, blood samples were obtained before bronchoscopy, and in the CAP group, after reaching stability. Plasma aliquots were withdrawn before neutrophil isolation for cytokine determination by ELISA. The isolation of neutrophils was performed as previously described [19]. The pellet was resuspended with a hypotonic solution for $5 \mathrm{~min}$ on ice, and spun down with PBS at $304 \times g$ for $5 \mathrm{~min}$ to pellet the neutrophils (similar to BAL samples). Cells were resuspended in PBS and counted.

\section{Detection of apoptosis by flow cytometry}

Neutrophils were stained with Annexin V detection kit (TACS $^{\mathrm{TM}}$ Annexin V-fluorescein isothiocyanate (FITC); R\&D Systems, Minneapolis, MN, USA) following the manufacturer's instructions. Samples were analysed using an EPICS XL (Beckman-Coulter, Hialea, FL, USA) [20]. Neutrophils were gated according to their forward and side scatter characteristics with a minimum of 10,000 gated events from each sample. Controls were included to set up compensation with no stained cells and cells stained with Annexin V or propidium iodide alone. Apoptotic cells were distinguished from normal and necrotic cells by labelling with Annexin $\mathrm{V}$ and exclusion of propidium iodide. Each subpopulation was expressed as a percentage of the total neutrophil population.

In addition, staining with CD16 FITC (IOTest; BeckmanCoulter) in separate aliquots of BAL and blood samples was applied to detect the positive neutrophil population.

For detection of apoptosis increments (spontaneous apoptosis) after $24 \mathrm{~h}$ of in vitro culture, neutrophils were seeded in RPMI supplemented with 10\% FBS, L-glutamine and antibiotics (penicillin and gentaminicin; Invitrogen) and cultured at $37^{\circ} \mathrm{C}$ for $24 \mathrm{~h}$.

\section{Measurement of cytokines IL-6, IL-8 and IL-10 in BAL and plasma}

The determination of IL-6, IL-8 and IL-10 was performed with a commercial enzymoimmunoassay technique (Pharmingen, BD Biociencias, Madrid, Spain) following the instructions of the manufacturer. The limits of detection were $4.7 \mathrm{pg} \cdot \mathrm{mL}^{-1}$ for IL-6, $3.1 \mathrm{pg} \cdot \mathrm{mL}^{-1}$ for IL-8, and $7.8 \mathrm{pg} \cdot \mathrm{mL}^{-1}$ for IL-10.

\section{Statistical analysis}

Statistical analysis was performed using the SPSS 12.0 and GraphPad software (San Diego, CA, USA). The Chi-squared test was used for categorical variables, and the Mann-Whitney U-test for continuous variables. Correlation was analysed using Spearman's rho correlation analysis. Data are shown as mean \pm SEM unless otherwise indicated. Cytokine levels and LOS are shown as medians and interquartile ranges (IQRs). A value of $p<0.05$ was considered statistically significant. The end-point variables were analysed with regard to the basal 
neutrophil apoptosis in BAL stratified as high $(\geqslant 20 \%$, 75th percentile of basal apoptosis) or low $(<20 \%)$ apoptosis rates.

\section{RESULTS}

\section{General characteristics of groups}

60 patients (29 NCAP, 15 CAP and 16 controls without infection) were recruited. Six NCAP patients were excluded due to an alternative diagnosis: one patient with tuberculosis, three with lung cancer, one with bronchiolitis obliterans and organising pneumonia, and one with invasive pulmonary aspergillosis, leaving 23 in the study group. Features of NCAP were: 11 patients with persistent fever and clinical worsening, nine with progression of infiltrates in chest radiograph (two with pleural effusion), and three with respiratory insufficiency requiring invasive ventilation; one of these patients died. The control group without infection included 16 adults, seven with minor haemoptysis and nine with peripheral lung nodules, whose diagnosis ruled out infection and whose comorbidities, sex and age were similar to those in the NCAP group and CAP group (table 1).

The median (IQR) interval since admission to the day in which samples were taken in the NCAP group was 6 (4-8) days, and was 5 (4-5) days in the CAP group.

Aetiological diagnosis in the NCAP group was established in $14(60.9 \%)$ cases: seven Streptococcus pneumoniae, one Escherichia coli, two methicillin-resistant Staphylococcus aureus (MRSA), one Pseudomonas aeruginosa and five mixed aetiology (three S. pneumoniae and other micro-organisms, two MRSA and other). In BAL fluid, the following micro-organisms were isolated: one S. pneumoniae, two Streptococcus spp., one Enterococcus spp.,

\begin{tabular}{|c|c|c|c|c|}
\hline \multirow[t]{2}{*}{ TABLE 1} & \multirow[b]{2}{*}{ NCAP } & \multirow[b]{2}{*}{ CAP } & \multirow[b]{2}{*}{ Control } & \multirow[b]{2}{*}{ p-value ${ }^{\#}$} \\
\hline & & & & \\
\hline Subjects $\mathrm{n}$ & 23 & 15 & 16 & \\
\hline Age yrs & $61 \pm 3$ & $66 \pm 4$ & $60 \pm 2$ & NS \\
\hline Male/female n & $16 / 7$ & $12 / 3$ & $14 / 2$ & NS \\
\hline Smoker/nonsmoker $\mathrm{n}$ & $6 / 17$ & $13 / 2$ & $9 / 7$ & NS \\
\hline \multicolumn{5}{|l|}{ Comorbidities } \\
\hline Heart disease & $6(26)$ & $3(20)$ & $2(12)$ & NS \\
\hline COPD & $7(30)$ & $7(46)$ & $5(31)$ & NS \\
\hline Chronic renal disease & $2(9)$ & 0 & $1(6)$ & NS \\
\hline Cerebrovascular disease & $3(13)$ & $3(20)$ & $1(6)$ & NS \\
\hline PSI score & $90 \pm 6$ & $102 \pm 7$ & 0 & \\
\hline $\begin{array}{l}\text { Blood leukocyte count } \\
\mathrm{mm}^{-3}\end{array}$ & $12752 \pm 1268$ & $9877 \pm 1065$ & $8217 \pm 730$ & 0.02 \\
\hline PMN \% & $82 \pm 11$ & $76 \pm 3$ & $62 \pm 4$ & 0.001 \\
\hline \multicolumn{5}{|l|}{ BAL fluid } \\
\hline Total cell count $\mathrm{mm}^{-3}$ & $7.5 \pm 2.2$ & & $0.5 \pm 0.2$ & 0.01 \\
\hline $\mathrm{PMN} \%$ & $38 \pm 4.5$ & & $8 \pm 3$ & $<0.001$ \\
\hline
\end{tabular}

Results are expressed as mean \pm SEM or $n(\%)$, unless otherwise stated. CAP: community-acquired pneumonia; COPD: chronic obstructive pulmonary disease; PSI: Pneumonia Severity Index (FINE score); PMN: polymorphonuclear neutrophils; BAL: bronchoalveolar lavage; NS: nonsignificant. ${ }^{\#}$ : MannWhitney U-test and Chi-squared test for continuous and categorical variables, respectively. one $P$. aeruginosa, two MRSA, one Acinetobacter baumannii, 10 potentially nonpathogenic micro-organisms, and none in three cases. Aetiology in the CAP group was: four S. pneumoniae (27\%), one $P$. aeruginosa $(7 \%)$ and unidentified in 10 cases.

\section{Apoptosis and cytokine results in BAL and blood}

Leukocyte counts in blood (table 1) and BAL fluid were significantly higher in the NCAP group. Apoptosis values from flow cytometry (fig. 1a-c) were compared with data obtained by judging the nuclear morphology by light microscopy (fig. 1d) and similar results were obtained.

The basal apoptosis in BAL (fig. 2) was higher (13 $\pm 3 \%$ ) in NCAP compared with controls $(6 \pm 3 \%)$, although significance was not reached; no data were available for CAP (as described in Methods and materials section). A similar pattern in blood was observed in NCAP compared with control and CAP $(15 \pm 4 \%, 4 \pm 1 \%$ and $6 \pm 2 \%$, respectively) (fig. $2 \mathrm{c}$ ). No significant correlation was found between apoptotic neutrophils in BAL and in blood in the NCAP group. Spontaneous apoptosis after $24 \mathrm{~h}$ of culture was studied in a subset of patients $(n=5)$ to observe neutrophil apoptosis in vitro (fig. $2 b$ and d). Spontaneous apoptosis was lower in blood in NCAP (compared with control and CAP; $\mathrm{p}<0.05)$. The same pattern appeared in BAL (compared with control, no data for CAP) although significance was not reached.

The median levels of systemic cytokines are depicted in figure 3. IL-6 was significantly higher in BAL of the NCAP patients (compared with controls), and in blood (compared with controls and a trend compared with CAP). IL-8 was higher in BAL in NCAP (compared with control), although no differences were found in blood (compared with controls and CAP). Conversely, IL-10 showed significantly higher levels in blood (compared with controls and CAP), without differences in BAL (compared with controls).

In the NCAP group, cytokine levels in BAL compared with blood showed significantly higher median (IQR) IL-8 BAL levels, $4,358(868-12,117)$ versus $38(24-78) \mathrm{pg} \cdot \mathrm{mL}^{-1}$, and significantly higher IL-10 in blood, 22 (9-60) versus 3 (2-9) pg. $\mathrm{mL}^{-1}$, whereas no differences were found concerning IL-6.

In the NCAP group, a statistically significant positive correlation was found between IL-6 in blood and BAL fluid ( $r=0.7$, $p=0.01$ ). IL-6 levels in BAL were also found to be significantly correlated to IL-10 levels in blood $(r=0.7, p=0.02)$. A significant correlation of the percentage and absolute count of neutrophils in BAL was found with IL-8 in BAL $(r=0.6, p=0.01$ and $r=0.7$, $\mathrm{p}<0.01$, respectively).

\section{Clinical resolution and outcome}

One of the NCAP patients died. The median (IQR) number of days to reach clinical stability was 12 (7-18) days, and the median LOS was 20 (12-34) days. Basal high neutrophil apoptosis in BAL $(\geqslant 20 \%)$ showed a significant negative correlation with LOS $(\mathrm{r}=-0.5, \mathrm{p}=0.02)$ and with days needed to reach clinical stability $(\mathrm{r}=-0.4, \mathrm{p}=0.05)$. Patients with high apoptosis in BAL had shorter LOS (median 10 versus 21 days) and needed fewer days for stability (median 7 versus 13 days). Neutrophil apoptosis in blood did not correlate either with clinical stability or with LOS $(\mathrm{r}=-0.2, \mathrm{p}=0.4$ and $\mathrm{r}=-0.1, \mathrm{p}=0.7$, respectively). 
a)

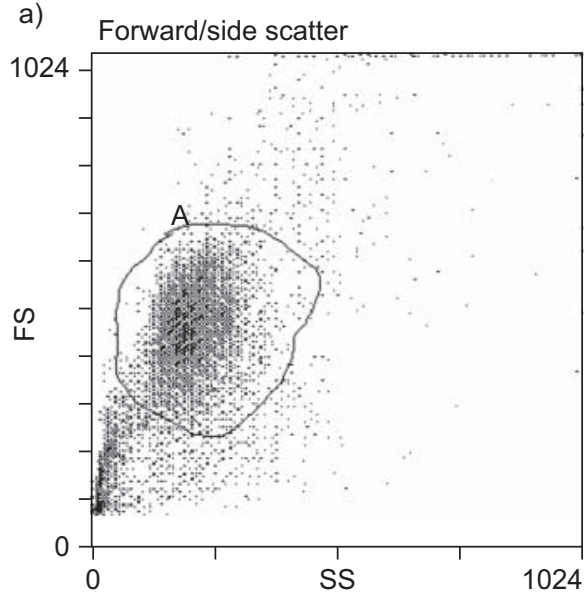

b)

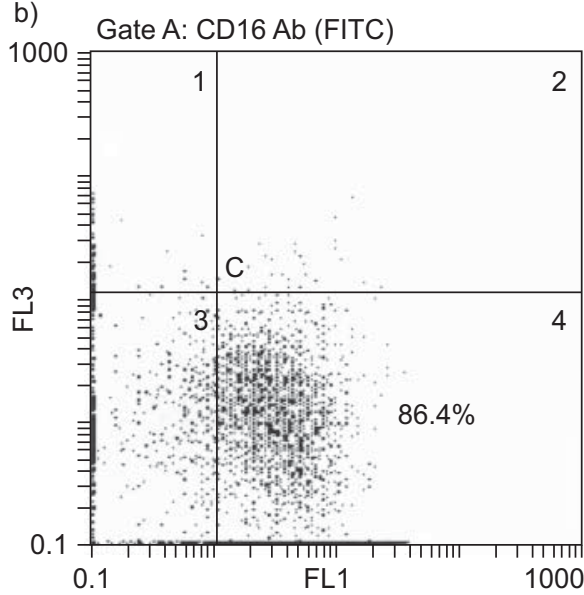

c)

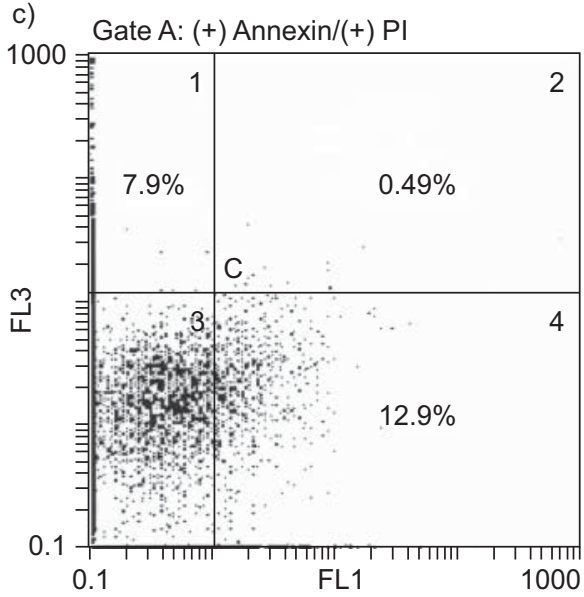

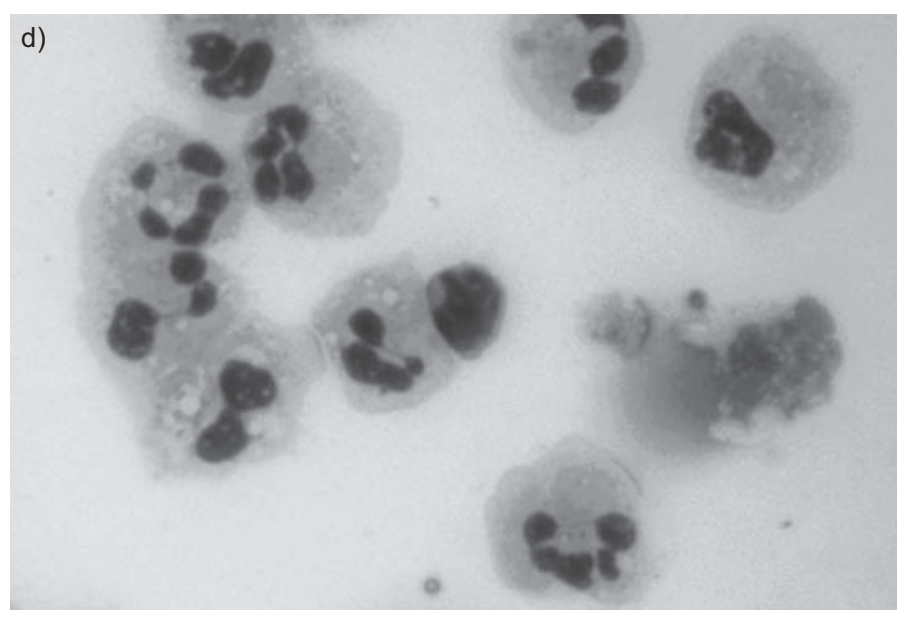

FIGURE 1. Flow cytometry of one representative bronchoalveolar lavage (BAL) sample. a) Neutrophils were gated according to their forward (FS) and side scatter (SS) characteristics. b) To confirm neutrophil population of gated area, cells were stained with CD16 antibody (fluorescein isothiocyanate (FITC)-conjugated). c) Gated area, containing neutrophils. Horizontal axis represents intensity of staining for Annexin V (FITC; logarithmic scale) and vertical axis intensity of staining for propidium iodide (PI; logarithmic scale). d) Light microscopy of BAL neutrophils in cytocentrifuge slides.
Cytokine levels in NCAP stratified according to high $(\geqslant 20 \%)$ or low $(<20 \%)$ basal BAL neutrophil apoptosis are depicted in figure 4. Systemic IL-10 was significantly lower in those with high apoptosis. A trend to lower IL-6 and IL-8 was found in those with high apoptosis both in BAL and blood. IL-6 and IL-10 levels in blood showed a positive significant correlation with the number of days to reach clinical stability $(r=0.7$, $\mathrm{p}<0.01$ and $\mathrm{r}=0.7, \mathrm{p}<0.01$, respectively), and IL-10 with LOS $(\mathrm{r}=0.7, \mathrm{p}=0.02)$. In the CAP group, a significant positive correlation was found for blood IL- 6 and IL-10 with number of days to reach clinical stability $(\mathrm{r}=0.4, \mathrm{p}=0.02$ and $\mathrm{r}=0.7$, $\mathrm{p}<0.001$, respectively), and with $\operatorname{LOS}(\mathrm{r}=0.4, \mathrm{p}=0.03$ and $\mathrm{r}=0.7, \mathrm{p}<0.001$, respectively).

\section{DISCUSSION}

The most important findings of this study are: 1) systemic basal neutrophil apoptosis was significantly higher in the NCAP group than in responding CAP and controls, while spontaneous apoptosis at $24 \mathrm{~h}$ was lower; 2 ) the inflammatory cytokine pattern showed higher pro-inflammatory IL-6 and IL-8 in BAL, and higher IL-6 along with higher IL-10 in blood; 3) patients with NCAP who had increased basal apoptosis in BAL $(\geqslant 20 \%)$ reached clinical stability earlier and their LOS was shorter; and 4) low basal alveolar apoptosis was associated with higher IL-10 in blood and a trend for higher IL-8 and IL-6 in BAL and blood.
The apoptosis of neutrophils after killing micro-organisms is considered essential for the downregulation of inflammation while precluding the release of their cytotoxic components. The relevance of high initial and persistent inflammation in CAP has been thoroughly studied. Initial raised inflammatory cytokines in NCAP, and at $72 \mathrm{~h}$, have been associated with slower clinical stability and poorer outcome [12, 21-23]. As expected, our findings showed an increase in cytokine levels and alveolar neutrophils, which reflects an ongoing inflammation along with certain degree of apoptosis. In fact, we found higher basal neutrophil apoptosis in BAL (12\%) than DROEMANN et al. [24] (1\%), probably because they included patients at an earlier stage. Interestingly, we also found a slightly higher neutrophil apoptosis in blood, reflecting the functional continuum of the infection.

Although absolute basal apoptosis was increased, neutrophil survival was also prolonged; that is, their functional longevity was enhanced. Spontaneous apoptosis at $24 \mathrm{~h}$ was higher in controls than in NCAP, as reported elsewhere [4]. This finding illustrates neutrophil fate during infection, especially because BAL neutrophils were not completely purified from the rest of the BAL cells, and cytokines released by co-cultured cells would reflect the in vivo inflammation process. Interestingly, in patients with clinical stability, both basal and spontaneous apoptosis mimics the pattern of controls without infection. The lower systemic basal apoptosis in CAP represents a phase in 
Apoptosis in BAL neutrophils
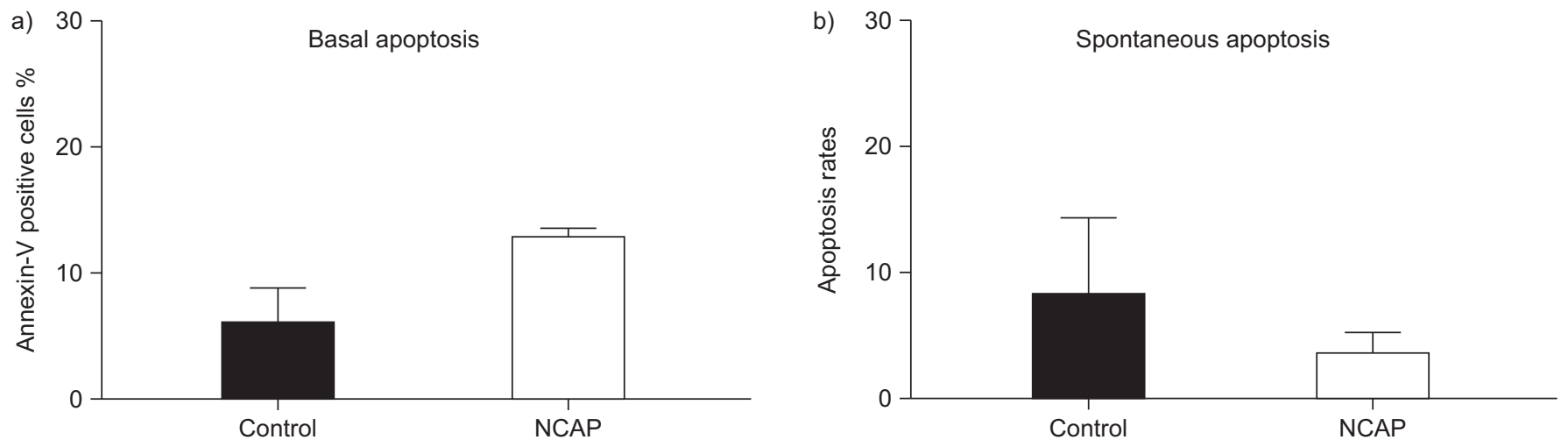

Apoptosis in blood neutrophils
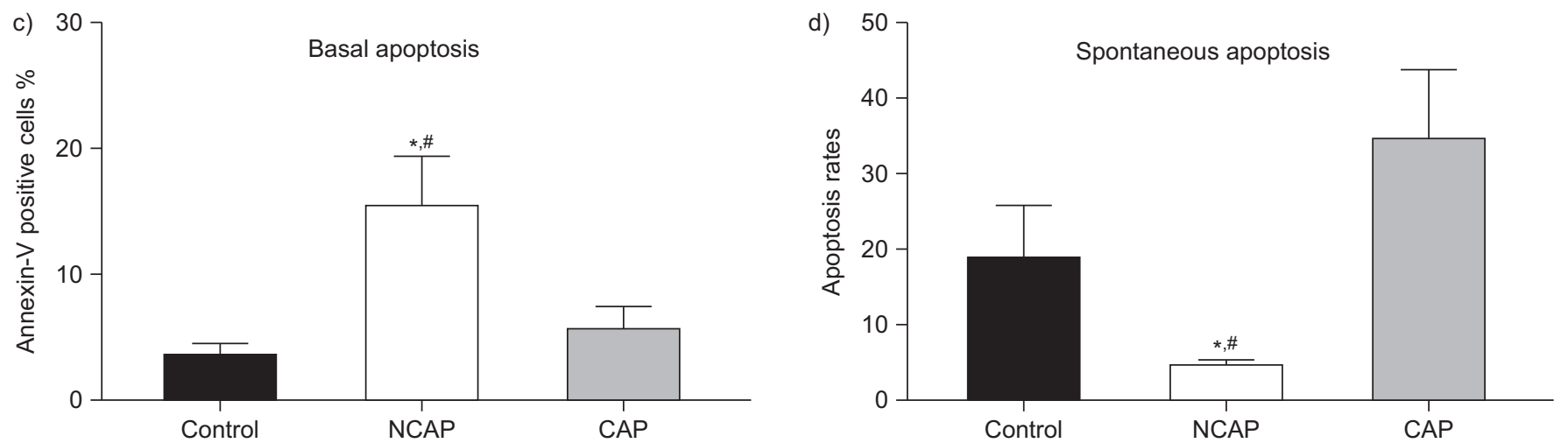

FIGURE 2. Apoptosis of neutrophils in bronchoalveolar lavage (BAL) of nonresponding community-acquired pneumonia (NCAP) and control (a and b) and peripheral blood of NCAP, control and community-acquired pneumonia (CAP) patients ( $\mathrm{c}$ and d). Apoptosis was determined after neutrophil purification (basal apoptosis, a and c) and after in vitro culture for $24 \mathrm{~h}$ (spontaneous apoptosis, $\mathrm{b}$ and d). Apoptosis rate is defined as percentage of cells in apoptosis at $24 \mathrm{~h}$ compared with that at $0 \mathrm{~h}$. Data are presented as mean \pm SEM. *: $p<0.05$, NCAP versus control; ${ }^{*}: p<0.05$, NCAP versus CAP.

which leukocytes are declining, as it corresponds to a resolving infection with decreasing cytokines (IL-6 and IL-10). These results indicate that a different neutrophil apoptosis kinetic is a feature of NCAP patients.

Activation of neutrophils is necessary to clear infection, as well as their apoptosis to adequately resolve inflammation [25]. During the initial phases of infection, cytokines may enhance neutrophil activation, particularly IL-8 prime neutrophils, and prolong their lifespan [26]. We have confirmed the higher proinflammatory cytokine levels of IL-6 and IL-8 in BAL fluid after $72 \mathrm{~h}$ of treatment, as expected in NCAP patients. Interestingly, the predominant inflammatory cytokines in the pulmonary and systemic compartments revealed different patterns: higher IL-8 in BAL, higher IL-10 in blood and similar IL-6 levels in both compartments. SCHÜTTE et al. [27] have also reported similar findings with IL-8 and IL- 6 in patients with severe pneumonia. The higher IL- 8 levels in BAL compared with blood highlight the active phase of infection and the upregulation of neutrophils. In fact, a positive correlation between IL- 8 and the absolute count of neutrophils in BAL was confirmed. Concerning IL-6, we found a positive correlation between levels in BAL and in blood, which indicates that an inflammatory process originating in the lung is continued in the systemic compartment. Conversely, IL-10 levels in blood indicate a predominant anti-inflammatory pattern. In fact, significantly lower systemic IL-10 and a trend for lower IL-6 was found in patients with clinical stability. Taking all these results together, a different speed of inflammatory resolution is suggested: slower in the lung than systemically. Moreover, we found a correlation between systemic inflammatory resolution and clinical resolution, i.e. among systemic IL-6 and IL-10 and stability and LOS.

The interaction between alveolar neutrophils, cytokines and adequate restoration of homeostasis is far from completely understood. Our results indicate that, when alveolar apoptosis was low in BAL, systemic IL-10 and IL-8 in BAL fluid were raised. This association may have several explanations. First, a reduced apoptosis may reflect an ongoing inflammation with the potential for prolonging the tissue damage. Secondly, bacterial load, or the pathogens themselves, might alter apoptosis thus evading their efficient killing [26]. Thirdly, an exuberant cytokine environment might delay apoptosis, as reported in acute lung injury, where it is associated with a dysregulation of apoptosis [28]. In sepsis, a delayed apoptosis due to prolonged activation of nuclear factor- $\kappa \mathrm{B}$ causes neutrophils to remain active $[29,30]$. Enhancement of neutrophil apoptosis, however, has 

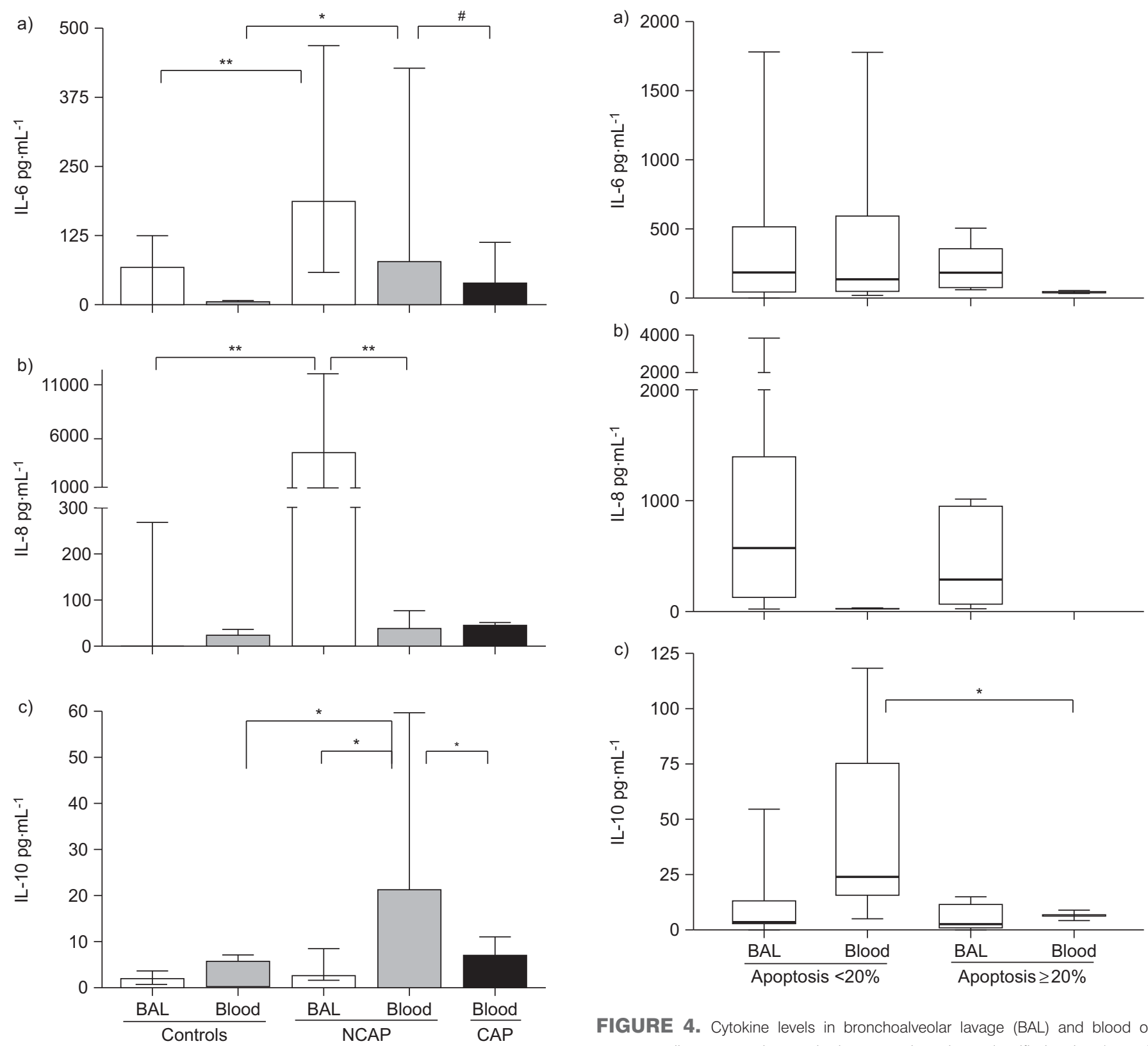

FIGURE 3. Cytokine levels from nonresponding community-acquired pneumonia (NCAP), community-acquired pneumonia (CAP) and control groups in bronchoalveolar lavage (BAL) and peripheral blood. Data are presented as median and interquartile range. ${ }^{*}: p<0.05 ;{ }^{*}: p<0.01 ;{ }^{*}: p=0.06$.

been associated with less lung damage and mortality [31]. In an animal model, localised apoptosis was found to be a feature in the resolution of pneumonia [32].

Clinical relevance of alveolar apoptosis was proved when analysing resolution of infectious parameters. We found that a higher alveolar apoptosis was significantly associated with faster clinical stability and shorter LOS. A similar significant correlation was found between systemic levels, both in NCAP and responding CAP, of cytokines, clinical stability and LOS.

To our knowledge, this is the first study to investigate the role of neutrophil apoptosis, both in the lung and systemically, in

FIGURE 4. Cytokine levels in bronchoalveolar lavage (BAL) and blood of nonresponding community-acquired pneumonia patients classified as low $(<20 \%)$ or high $(\geqslant 20 \%)$ BAL apoptosis. Data are presented as median and interquartile range. *: $p<0.05$.

NCAP. With regard to potential limitations, we have to point out a possible beta error in statistical analyses due to the limited number of cases included and the lack of BAL in the responding $\mathrm{CAP}$ group.

In summary, basal apoptosis in NCAP is high both in lung and in blood while spontaneous apoptosis at $24 \mathrm{~h}$ is lower than in responding CAP. High IL-8 levels in BAL fluid were associated with accumulation of neutrophils in the lung. Systemic inflammation with raised IL-6 and IL-10 is associated with a delayed clinical stability and LOS. In NCAP, a greater basal alveolar neutrophil apoptosis was associated with earlier clinical stability and lower LOS, which suggests that neutrophil apoptosis precludes persistent local inflammation, thereby contributing to a faster recovery of normal homeostasis. 


\section{SUPPORT STATEMENT}

This work was supported by the CIBER de Enfermedades Respiratorias (CIBERES), an initiative from the Carlos III Health Institute, Grant from Bancaja-Fundación para la Investigación Hospital La Fe, Conselleria de Sanidad de la Comunidad Valenciana (AP-018/06) and SAF2009-08913.

\section{STATEMENT OF INTEREST}

None declared.

\section{REFERENCES}

1 Guidelines for the diagnosis and treatment of communityacquired pneumonia. Spanish Society of Pulmonary and Thoracic Surgery (SEPAR). Arch Bronconeumol 2005; 41: 272-289.

2 Mitzgerd JP, Lupa MM, Kogan MS, et al. Nuclear factor-кB p50 limits inflammation and prevents lung injury during Escherichia coli pneumonia. Am J Respir Crit Care Med 2003; 168: 810-817.

3 Menéndez R, Perpiñá M, Torres $\mathrm{A}$, et al. Evaluation of nonresolving and progressive pneumonia. Semin Respir Infect 2003; 18: 103-111.

4 Pletz MW, Ioanas M, de Roux A, et al. Reduced spontaneous apoptosis in peripheral blood neutrophils during exarcerbation of COPD. Eur Respir J 2004; 23: 532-537.

5 Behnia M, Robertson KA, Martin WJ 2nd, et al. Lung infections: role of apoptosis in host defense and pathogenesis of disease. Chest 2000; 117: 1771-1777.

6 François S, El Benna J, Dang PM, et al. Inhibition of neutrophil apoptosis by TLR agonists in whole blood: involvement of the phosphoinositide 3-kinase/Akt and NF-kB signaling pathways, leading to increased levels of Mcl-1, A1, and phosphorylated Bad. J Immunol 2005; 174: 3633-3642.

7 Cowburn AS, Condliffe AM, Farahi N, et al. Advances in neutrophil biology: clinical implications. Chest 2008; 134: 606-612.

8 Watt AP, Brown V, Courtney J, et al. Neutrophil apoptosis, proinflammatory mediators and cell counts in bronchiectasis. Thorax 2004; 59: 231-236.

9 Knapp S, Leemans JC, Florquin S, et al. Alveolar macrophages have a protective antiinflammatory role during murine pneumococcal pneumonia. Am J Respir Crit Care Med 2003; 167: 171-179.

10 Montón C, Torres A, El-Ebiary M, et al. Cytokine expression in severe pneumonia: a bronchoalveolar lavage study. Crit Care Med 1999; 27: 1745-1753.

11 Ioanas M, Ferrer M, Cavalcanti M, et al. Causes and predictors of nonresponse to treatment of intensive care unit-acquired pneumonia. Crit Care Med 2004; 32: 938-945.

12 Menéndez R, Cavalcanti M, Reyes S, et al. Markers of treatment failure in hospitalised community acquired pneumonia. Thorax 2008; 63: 447-452.

13 Fine MJ, Auble TE, Yealy DM, et al. A prediction rule to identify low-risk patients with community-acquired pneumonia. $N$ Engl J Med 1997; 336: 243-250.

14 Halm EA, Fine MJ, Marrie TJ, et al. Time to clinical stability in patients hospitalized with community-acquired pneumonia: implications for practice guidelines. JAMA 1998; 279: 1452-1457.

15 Menéndez R, Torres A, Rodríguez de Castro F, et al. Neumofail Group. Reaching stability in community-acquired pneumonia: the effects of the severity of disease, treatment, and the characteristics of patients. Clin Infect Dis 2004; 39: 1783-1790.

16 Technical recommendations and guidelines for bronchoalveolar lavage. Report of the European Society of Pneumology Task Group. Eur Respir J 1989; 2: 561-585.

17 Ishii H, Mukae H, Kadota J, et al. Increased levels of interleukin-18 in bronchoalveolar lavage fluid of patients with idiopathic nonspecific interstitial pneumonia. Respiration 2005; 72: 39-45.

18 Hodge S, Hodge G, Scicchitano R, et al. Alveolar macrophages from subjects with chronic obstructive pulmonary disease are deficient in their ability to phagocytose apoptotic airway epithelial cells. Immunol Cell Biol 2003; 81: 289-296.

19 Ottonello L, Dapino P, Amelotti M, et al. Activation of neutrophil respiratory burst by cytokines and chemoattractants. Regulatory role of extracellular matrix glycoproteins. Inflamm Res 1998; 47: 345-350.

20 Schmidt-Ioanas M, Pletz MW, de Roux A, et al. Apoptosis of peripheral blood neutrophils in COPD exacerbation does not correlate with serum cytokines. Respir Med 2006; 100: 639-647.

21 Kellum JA, Kong L, Fink MP, et al. GenIMS Investigators. Understanding the inflammatory cytokine response in pneumonia and sepsis: results of the Genetic and Inflammatory Markers of Sepsis (GenIMS) Study. Arch Intern Med 2007; 167: 1655-1663.

22 Chalmers JD, Singanayagam A, Hill AT, et al. C-reactive protein is an independent predictor of severity in community-acquired pneumonia. Am J Med 2008; 121: 219-225.

23 Menéndez R, Martínez R, Reyes S, et al. Stability in communityacquired pneumonia. One step forward with markers? Thorax 2009; 64: 987-992.

24 Droemann D, Aries SP, Hansen F, et al. Decreased apoptosis and increased activation of alveolar neutrophils in bacterial pneumonia. Chest 2000; 117: 1679-1684.

25 Wesche DE, Lomas-Neira JL, Perl M, et al. Leukocyte apoptosis and its significance in sepsis and shock. J Leukoc Biol 2005; 78: 325337.

26 DeLeo FR. Modulation of phagocyte apoptosis by bacterial pathogens. Apoptosis 2004; 9: 399-413.

27 Schütte H, Lohmeyer J, Rosseau S, et al. Bronchoalveolar and systemic cytokine profiles in patients with ARDS, severe pneumonia and cardiogenic pulmonary oedema. Eur Respir J 1996; 9: 1858-1867.

28 Chopra M, Reuben JS, Sharma AC. Acute lung injury: apoptosis and signalling mechanisms. Exp Biol Med 2009; 234: 361-371.

29 Taneja R, Parodo J, Jia SH, et al. Delayed neutrophil apoptosis in sepsis is associated with maintenance of mitochondrial transmembrane potential and reduced caspase-9 activity. Crit Care Med 2004 32: $1460-1469$.

30 Sayeed MM. Delay of neutrophil apoptosis can exacerbate inflammation in sepsis patients: cellular mechanisms. Crit Care Med 2004; 32: 1604-1606.

31 Sookhai S, Wang JJ, McCourt M, et al. A novel therapeutic strategy for attenuating neutrophil-mediated lung injury in vivo. Ann Surg 2002; 235: 285-291.

32 Kazzaz JA, Horowitz S, Xu J, et al. Differential patterns of apoptosis in resolving and nonresolving bacterial pneumonia. Am J Respir Crit Care Med 2000; 161: 2043-2050. 